\title{
A NOTE ON DERIVED MCKAY CORRESPONDENCE
}

\author{
Jiun-Cheng Chen and Hsian-Hua Tseng
}

\begin{abstract}
We obtain a global version and a twisted version (in the sense of [BP05]) of the main theorem of [BKR01].
\end{abstract}

\section{Introduction}

We work over the field of complex numbers.

Let $X$ be an irreducible projective variety of dimension $n$. Assume that $X$ has only quotient singularities. According to [Vi89], there is a smooth Deligne-Mumford stack $\mathcal{X}$ with coarse moduli space $X$, such that $\mathcal{X}$ and $X$ are isomorphic in codimension one. Note that $\mathcal{X}$ is a quotient stack. Let $\pi: \mathcal{X} \rightarrow X$ denote the projection.

In the case where $X=M / G$ with $G$ a finite group, let $Y \subset \mathrm{G}-\mathrm{Hilb}(M)$ be the irreducible component of the $G$-Hilbert scheme of $M$ that contains the free orbits. There is a morphism $Y \rightarrow X$ given by the Hilbert-Chow morphism. The main result of [BKR01] can be stated as follows: Suppose that $\operatorname{dim} Y \times_{X} Y \leq n+1$, then $Y$ is smooth and there is an equivalence of derived categories $D^{b}(Y) \simeq D^{b}(\mathcal{X})$.

We study the global version of this problem (that is, $X$ is not necessarily of the form $M / G$ as above). Recall that for any Deligne-Mumford stack $\mathcal{X}$, étale locally on its coarse moduli space, $\mathcal{X}$ is of the form $[U / G]$ with $U$ a scheme and $G$ a finite group. It is tempting to obtain a global crepant resolution by patching the local ones. This is, however, not obvious at all: Suppose that $\left\{V_{i}\right\}$ is an étale cover of $X$, $V_{i} \times_{X} \mathcal{X} \simeq\left[U_{i} / G_{i}\right]$, and for each $i$ there is a crepant resolution $\phi_{i}: Y_{i} \rightarrow V_{i}$. Then it is not clear that we can patch these $\left\{Y_{i}\right\}$ together, since crepant resolution in higher dimension is not unique. In dimension 3 , a global crepant resolution can be built from local ones since flops preserve smoothness and two crepant resolutions can be connected by a sequence of flops, see Proposition 3.4 below. In higher dimensions, this argument doesn't work since flops do not preserve smoothness and may not terminate.

An observation, which we learned from D. Abramovich, is that a certain Hilbert functor studied by Olsson-Starr [OS03] is a good replacement for $G$-Hilbert schemes in the global situation. We denote the scheme representing this Hilbert functor by $\operatorname{Hilb}(\mathcal{X})$. We will only be interested in a particular component $\operatorname{Hilb}^{\prime}(\mathcal{X}) \subset \operatorname{Hilb}(\mathcal{X})$ : Let $U \subset \mathcal{X}$ be the open set of non-stacky points in $\mathcal{X}$. There is a natural inclusion $U \subset \operatorname{Hilb}(\mathcal{X})$. The scheme $\operatorname{Hilb}^{\prime}(\mathcal{X})$ is the component which contains $U$. There is a

Received by the editors December 13, 2006. Revision received March 8, 2008.

Research of J.-C. C supported in part by National Science Council grant 95-2115-M-007-018 and National Center of Theoretical Science in Hsinchu, Taiwan and by the Golden Jade Fellowship of Kenda Foundation.

Research of H.-H. T supported in part by Clay Mathematics Institute Liftoff Fellowship program. 
morphism

induced by the functor $\pi_{*}$.

$$
\operatorname{Hilb}^{\prime}(\mathcal{X}) \rightarrow X
$$

Theorem 1.1. Assume that $\operatorname{dimHilb}^{\prime}(\mathcal{X}) \times_{X} \operatorname{Hilb}^{\prime}(\mathcal{X}) \leq n+1$, then $\operatorname{Hilb}^{\prime}(\mathcal{X})$ is smooth and the Fourier-Mukai type transformation

$$
F: D^{b}\left(H i l b^{\prime}(\mathcal{X})\right) \rightarrow D^{b}(\mathcal{X})
$$

induced by the universal object over $\operatorname{Hilb}^{\prime}(\mathcal{X})$ is an equivalence of derived categories.

Remark 1.2. Crepant resolutions, if exist, may not be unique. In [BKR01] a particular crepant resolution is constructed as a moduli space. It is expected that this is a general phenomenon: every crepant resolution can be constructed as a moduli space. This is proved in [CI05] when $X=\mathbb{C}^{3} / G$ where $G$ is abelian. We speculate that the same is true for global orbifolds: every crepant resolution of a global orbifold can be constructed as a moduli space. Some variants of the Quot functor may be helpful. We hope to return to this problem in the future.

Remark 1.3. Consider the case when $X$ has only symplectic quotient singularities. If $\operatorname{Hilb}^{\prime}(\mathcal{X}) \rightarrow X$ is a crepant resolution, then we have an equivalence of derived categories $D^{b}(\mathcal{X}) \rightarrow D^{b}\left(\operatorname{Hilb}^{\prime}(\mathcal{X})\right.$, cf [Kal05].

In [BP05], a conjectural twisted version of derived McKay correspondence is formulated. This conjecture suggests an equivalence between the derived category of twisted sheaves on an orbifold and a related derived category of twisted sheaves on a crepant resolution. We establish this conjecture in the setting of [BKR01], see Theorem 4.1.

1.1. Differential graded categories. It is known (e.g. [BK90]) that the derived category $D(X)$ of coherent sheaves can be enriched to a differential graded (DG) category which we denote by $L_{c o h}(X)$. Roughly speaking, objects in $L_{c o h}(X)$ are complexes of sheaves. For two objects $A=\left(A^{*}\right)$ and $B=\left(B^{*}\right)$, a morphism $\phi$ of degree $i$ between them is a collection $\left(\phi^{*}\right), \phi^{*}: A^{*} \rightarrow B^{+i}$. The space $\operatorname{Hom}_{D G}(A, B)$ of morphisms is a $\mathbb{Z}$-graded vector space endowed with a differential $d \phi=d_{B}$ 。 $\phi-(-1)^{i} \phi \circ d_{A}$. In other words, $\operatorname{Hom}_{D G}$ is a complex. One recovers $D(X)$ by localizing the homotopy category of $L_{c o h}(X)$ with respect to the subcategory of acyclic complexes. For more details, see [Ke99].

Here we add a note to the theory of D-equivalence that Fourier-Mukai type equivalence can be "lifted" to the level of DG-categories.

Lemma 1.4. Let $F: L_{c o h}(X) \rightarrow L_{c o h}(Y)$ be a $D G$ functor which induces an equivalence of derived categories $D(X) \rightarrow D(Y)$. Then $F$ is a quasi-equivalence of $D G$ categories.

Proof. Recall that a quasi-equivalence is a DG functor $F: L_{\text {coh }}(X) \rightarrow L_{\text {coh }}(Y)$ such that

(1) The morphism $\operatorname{Hom}_{D G}(A, B) \rightarrow \operatorname{Hom}_{D G}(F A, F B)$ is a quasi-equivalence of complexes;

(2) $F$ induces an equivalence $D(X) \rightarrow D(Y)$ of triangulated categories. 
Hence we only need to show the first condition, i.e. the cohomologies coincide. In degree zero, this is part of the derived equivalence:

$$
H^{0} \operatorname{Hom}_{D G}(A, B) \simeq H^{0} \operatorname{Hom}_{D G}(F A, F B) .
$$

On the other hand, we have

$$
\begin{gathered}
H^{i} \operatorname{Hom}_{D G}(A, B)=H^{0} \operatorname{Hom}_{D G}(A, B[i]) \\
\simeq H^{0} \operatorname{Hom}_{D G}(F A, F B[i])=H^{i} H_{D G}(F A, F B) .
\end{gathered}
$$

This completes the proof.

Since pushforward, pullback, and tensor product functors can be lifted to DG level, it is clear that a Fourier-Mukai functor comes from a DG functor $L_{c o h}(X) \rightarrow L_{c o h}(Y)$ which, according to the lemma above, is a quasi-equivalence. It's clear from the proof that in this context, having a quasi-equivalence at DG level yields no new information. However, the DG structure is sometimes necessary: for example, to construct the Bmodel potential of a Calabi-Yau manifold using a recent work of K. Costello [Co05].

This paper is organized as follows. Several properties of the Quot functors we need are established in Section 2. Theorem 1.1 is proved in Section 3. In Section 4 we prove some cases of a conjecture in [BP05] concerning twisted derived McKay correspondence.

\section{Acknowledgements}

We thank D. Abramovich, V. Baranovsky, D. Ben-Zvi, B. Keller, M. Olsson, and J. Starr, for many important discussions. Part of this work was done during the AMS summer institute in algebraic geometry in Seattle. We thank the organizers for hospitality and support.

\section{Quot functors after Olsson-Starr}

In this section we discuss some properties of Quot functors for Deligne-Mumford stacks following Olsson-Starr [OS03]. We will focus on the case of quotients of $\mathcal{O}_{\mathcal{X}}$. Let $\operatorname{Quot}\left(\mathcal{O}_{\mathcal{X}} / \mathcal{X}\right)\left(\right.$ respectively $\left.\operatorname{Quot}\left(\mathcal{O}_{X} / X\right)\right)$ denote the Quot functor associated to the sheaf $\mathcal{O}_{\mathcal{X}}$ over $\mathcal{X}$ (respectively the sheaf $\mathcal{O}_{X}$ over $X$ ). According to [Gr62] and [OS03], these two functors are representable by projective schemes which we denote by $\operatorname{Quot}\left(\mathcal{O}_{\mathcal{X}} / \mathcal{X}\right)$ and $\operatorname{Quot}\left(\mathcal{O}_{X} / X\right)$ respectively.

Definition 2.1. There is a morphism $\operatorname{Quot}\left(\mathcal{O}_{\mathcal{X}} / \mathcal{X}\right) \rightarrow \operatorname{Quot}\left(\mathcal{O}_{X} / X\right)$ defined as follows: The exact functor $\pi_{*}: \operatorname{Coh}(\mathcal{X}) \rightarrow \operatorname{Coh}(X)$ yields a natural transformation $\operatorname{Quot}\left(\mathcal{O}_{\mathcal{X}} / \mathcal{X}\right) \Rightarrow \operatorname{Quot}\left(\mathcal{O}_{X} / X\right)$ of Quot functors. Let $\operatorname{Quot}\left(\mathcal{O}_{\mathcal{X}} / \mathcal{X}\right) \rightarrow \operatorname{Quot}\left(\mathcal{O}_{X} / X\right)$ be the induced morphism between the corresponding schemes.

Note that $X \subset \operatorname{Quot}\left(\mathcal{O}_{X} / X\right)$ is an irreducible component. Let $\operatorname{Hilb}^{\prime}(\mathcal{X}) \subset$ Quot $\left(\mathcal{O}_{\mathcal{X}} / \mathcal{X}\right)$ denote the irreducible component containing the preimage of $X_{s m}$. Restricting the morphism $\operatorname{Quot}\left(\mathcal{O}_{\mathcal{X}} / \mathcal{X}\right) \rightarrow \operatorname{Quot}\left(\mathcal{O}_{X} / X\right)$ yields the morphism $\operatorname{Hilb}^{\prime}(\mathcal{X}) \rightarrow X$. Note that $\operatorname{Hilb}^{\prime}(\mathcal{X})$ is contained in the locus $\operatorname{Quot}^{1}\left(\mathcal{O}_{\mathcal{X}} / \mathcal{X}\right)$ which parametrizes quotients with Hilbert polynomial 1. 
Lemma 2.2. Assume that $\mathcal{X}$ is of the form $[M / G]$ with $M$ quasi-projective and $G$ finite. Then $\operatorname{Hilb}^{\prime}(\mathcal{X})$ is isomorphic to the irreducible component of $G$-Hilb(M) containing the free $G$-orbits.

Proof. There is a natural morphism $\operatorname{Hilb}^{\prime}(\mathcal{X}) \rightarrow$ G-Hilb $(M)$ defined as follows: Given an object $\mathcal{Z} \subset \mathcal{X} \times S$ of $H_{i l b^{\prime}}(\mathcal{X})(S)$. The scheme

$$
\mathcal{Z} \times{ }_{(\mathcal{X} \times S)}(M \times S) \subset M \times S
$$

is naturally an $S$-family of $G$-clusters: Clearly the group scheme $G \times S$ acts fiberwise on $\mathcal{Z} \times \mathcal{X} \times S(M \times S)$. Also, since $M \times S \rightarrow \mathcal{X} \times S$ is a principle $G$-bundle, the space $\Gamma\left(\mathcal{O}_{\mathcal{Z} \times \mathcal{X} \times S}(M \times S)\right)$ coincides with the regular representation $\mathbb{C}[G] \otimes \Gamma\left(\mathcal{O}_{S}\right)$ of $G \times S$. Thus this defines an object in G-Hilb $(M)(S)$.

It is easy to check that this morphism is a closed immersion, as one may recover $\mathcal{Z} \subset$ $\mathcal{X} \times S$ by taking the stack quotient $[\mathcal{Z} \times \mathcal{X} \times S(M \times S) / G]$. Moreover, let G-Hilb $(M) \rightarrow$ $X=M / G$ be the morphism induced from the Hilbert-Chow morphism. Then the following diagram commutes:

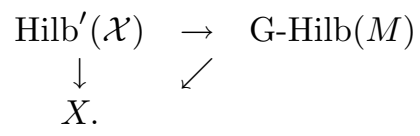

The result follows.

An important property is that $\operatorname{Hilb}^{\prime}(\mathcal{X})$ behaves well under étale base-change on the coarse moduli space.

Proposition 2.3. Let $U \rightarrow X$ be an étale morphism from a scheme $U$ such that $U$ is finite to its image. Then we have

$$
U \times_{X} \operatorname{Hilb}^{\prime}(\mathcal{X}) \simeq \operatorname{Hilb}^{\prime}\left(U \times_{X} \mathcal{X}\right) .
$$

Proof. First note that we may identify a scheme with the Hilbert scheme of a point on it. In particular, we have $\operatorname{Hilb}^{1}(U) \simeq U$ and $\operatorname{Hilb}^{1}(X) \simeq X$.

Consider the diagram

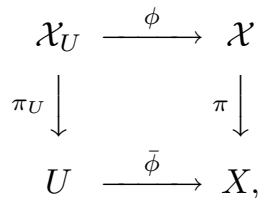

where $\mathcal{X}_{U} \cong U \times_{X} \mathcal{X}$. Note that $U$ is a coarse moduli space of $U \times_{X} \mathcal{X}$.

We will define two morphisms $\alpha: U \times_{X} \operatorname{Hilb}^{\prime}(\mathcal{X}) \rightarrow \operatorname{Hilb}^{\prime}\left(U \times_{X} \mathcal{X}\right)$ and $\beta$ : $\operatorname{Hilb}^{\prime}\left(U \times_{X} \mathcal{X}\right) \rightarrow U \times_{X} \operatorname{Hilb}^{\prime}(\mathcal{X})$.

Definition of $\alpha$ : Consider any morphism $f: S \rightarrow U \times_{X} \operatorname{Hilb}^{\prime}(\mathcal{X})$. By the universal property of the fiber product, the morphism $f: S \rightarrow U \times_{X} \operatorname{Hilb}^{\prime}(\mathcal{X})$ corresponds to three objects

$$
\begin{gathered}
{\left[\mathcal{O}_{U \times S} \rightarrow P\right] \in \operatorname{Hilb}^{1}(U)(S),\left[\mathcal{O}_{\mathcal{X} \times S} \rightarrow Q\right] \in \operatorname{Hilb}^{\prime}(\mathcal{X})(S)} \\
\text { and }\left[\mathcal{O}_{X \times S} \rightarrow R\right] \in \operatorname{Hilb}^{1}(X)(S),
\end{gathered}
$$

such that the quotients

$$
(\pi \times i d)_{*} \mathcal{O}_{\mathcal{X} \times S} \rightarrow(\pi \times i d)_{*} Q, \text { and } \mathcal{O}_{X \times S} \rightarrow(\bar{\phi} \times i d)_{*} \mathcal{O}_{U \times S} \rightarrow(\bar{\phi} \times i d)_{*} P
$$


coincide with $\mathcal{O}_{X \times S} \rightarrow R$. We can identify these objects with

Consider the corresponding morphism $h_{1}: S \rightarrow U$ (resp. $\left.h_{2}: S \rightarrow X\right)$ of $\left[\mathcal{O}_{U \times S} \rightarrow\right.$ $P] \in \operatorname{Hilb}^{1}(U)(S)\left(\operatorname{resp} .\left[\mathcal{O}_{X \times S} \rightarrow R\right] \in \operatorname{Hilb}^{1}(X)(S)\right)$.

Consider the quotient

$$
\mathcal{O}_{U \times X \times} \mathcal{X} \times S \rightarrow\left(\pi_{U} \times i d\right)^{*} P \otimes_{(\phi \times i d) *(\pi \times i d) * R}(\phi \times i d)^{*} Q .
$$

It is straightforward to check that this gives an object in $H_{i l b}\left(U \times_{X} \mathcal{X}\right)(S)$, thus defines the morphism $\alpha$. We give a more geometric interpretation of the map $\alpha$ : Let $\mathcal{Z}_{X, S} \subset S \times \mathcal{X}$ be the correspodening substack. Consider the substack $(\phi \times$ $i d)^{-1} \mathcal{Z}_{X, S} \subset S \times U \times_{X} \mathcal{X}$. Note that the coarse space of $\mathcal{Z}_{X, S}$ is $S$ and the coarse space of $(\phi \times i d)^{-1} \mathcal{Z}_{X, S}$ is $S \times{ }_{X} U$, which contains $S$ as a component (recall that we have the morphism $S \rightarrow U$ ). Pulling back the stack $(\phi \times i d)^{-1}\left(\mathcal{Z}_{X, S}\right)$ (over $U$ ) to $S$ via the morphism $S \rightarrow U$. The resulting stack in proper over $S$.

The stack $(\phi \times i d)^{-1}\left(\mathcal{Z}_{X, S}\right)$ is not the stack we need. Tensoring the structure sheaf of $(\phi \times i d)^{-1}\left(\mathcal{Z}_{X, S}\right)$ with $\left.\pi_{U} \times i d\right)^{*} P$, i.e. pulling back to $S$ via the morphism $S \rightarrow U$, gives the correct substack.

Definition of $\beta$ : Given an object $\mathcal{O}_{U \times_{X} \mathcal{X} \times S} \rightarrow P$ of $H i l b\left(U \times_{X} \mathcal{X}\right)(S)$. Let $\mathcal{Z}_{U, S} \subset S \times\left(U \times{ }_{X} \mathcal{X}\right)$ be the corresponding substack. Note that $(\phi \times i d)_{*} \mathcal{Z}_{U, S} \simeq S$ and $\left.(\phi \times i d)\right|_{\mathcal{Z}_{U, S}}: \mathcal{Z}_{U, S} \rightarrow \mathcal{X} \times S$ is proper.

We claim that the morphism $\left.(\phi \times i d)\right|_{\mathcal{Z}_{U, S}}: \mathcal{Z}_{U, S} \rightarrow \mathcal{X} \times S$ is isomorphic to its image in $\mathcal{X} \times S$. This is local on $S$. Let $h_{1}: S \rightarrow X$. Consider a étale altas $g: X_{1} \rightarrow X$ May assume that the image $h_{1}(S)$ lies in $g\left(X_{1}\right)$ and $g: X_{1} \rightarrow X$ is finite (to its image) of degree $d$.Consider the pull back of $X_{1}$ to $U$. The morphism $X_{2} \rightarrow U$ is also finite to its image.

This can be check locally on $S$. Shrinking $U$ if necessary, we may assume that $U \rightarrow \bar{\phi}$ is finite, say of degree $d$. Consider the stack

Therefore the map $(\phi \times i d)_{*} \mathcal{O}_{U \times x_{X} \mathcal{X} \times S} \rightarrow(\phi \times i d)_{*} P$ is surjective and the composite

$$
\mathcal{O}_{\mathcal{X} \times S} \rightarrow(\phi \times i d)_{*} \mathcal{O}_{U \times X} \mathcal{X} \times S \rightarrow(\phi \times i d)_{*} P
$$

is surjective. Since $\mathcal{Z}_{S} \rightarrow(\phi \times i d)\left(\mathcal{Z}_{S}\right)$ is an isomorphism, $(\phi \times i d)_{*} P$ is proper over $S$.

Hence we obtain an object $\mathcal{O}_{\mathcal{X} \times S} \rightarrow(\phi \times i d)_{*} P$ of $\operatorname{Hilb}^{\prime}(\mathcal{X})(S)$. This defines a morphism

$$
H i l b^{\prime}\left(U \times_{X} \mathcal{X}\right) \rightarrow H i l b^{\prime}(\mathcal{X}) .
$$

The functor $\pi_{U *}$ induces a morphism $\operatorname{Hilb}^{\prime}\left(U \times_{X} \mathcal{X}\right) \rightarrow U$. It follows from the equality $\pi \circ \phi=\bar{\phi} \circ \pi_{U}$ that the two composites $\operatorname{Hilb}^{\prime}\left(U \times_{X} \mathcal{X}\right) \rightarrow \operatorname{Hilb}^{\prime}(\mathcal{X}) \rightarrow X$ and $\operatorname{Hilb}^{\prime}\left(U \times_{X} \mathcal{X}\right) \rightarrow U \stackrel{\bar{\phi}}{\rightarrow} X$ coincide. Therefore we obtain a morphism $\beta$ : $\operatorname{Hilb}^{\prime}\left(U \times_{X} \mathcal{X}\right) \rightarrow U \times_{X} \operatorname{Hilb}^{\prime}(\mathcal{X})$.

To conclude, it suffices to show that both $\alpha \circ \beta$ and $\beta \circ \alpha$ are identity functors. We begin with $\beta \circ \alpha$. Consider a morphism $S \rightarrow U \times{ }_{X} \operatorname{Hilb}^{\prime}(\mathcal{X})$ which corresponds to three objects described above. Since

$$
\begin{gathered}
(\phi \times i d)_{*}\left(\left(\pi_{U} \times i d\right)^{*} P \otimes_{(\phi \times i d)^{*}(\pi \times i d)^{*} R}(\phi \times i d)^{*} Q\right) \\
\simeq(\pi \times i d)^{*}(\bar{\phi} \times i d)_{*} P \otimes_{(\pi \times i d)^{*} R} Q \simeq Q \\
\left(\pi_{U} \times i d\right)_{*}\left(\left(\pi_{U} \times i d\right)^{*} P \otimes_{(\phi \times i d) *(\pi \times i d)^{*} R}(\phi \times i d)^{*} Q\right) \\
\simeq P \otimes_{(\bar{\phi} \times i d)^{*} R}(\bar{\phi} \times i d)^{*}(\pi \times i d)_{*} Q \simeq P .
\end{gathered}
$$


It follows that $\beta \circ \alpha$ is the identity functor.

It remains to prove that $\alpha \circ \beta$ is the identity functor. Note that for this we may assume that $U$ is a formal neighborhood of a point in $X$. Consider an object $\mathcal{O}_{U \times X_{X} \mathcal{X} \times S} \rightarrow P$ of $H_{i l b^{\prime}}\left(U \times_{X} \mathcal{X}\right)(S)$. Applying $\beta$ yields two objects

$$
\left[\mathcal{O}_{\mathcal{X} \times S} \rightarrow(\phi \times i d)_{*} P\right] \in \operatorname{Hilb}^{\prime}(\mathcal{X})(S) \text { and }\left[\mathcal{O}_{U \times S} \rightarrow\left(\pi_{U} \times i d\right)_{*} P\right] \in \operatorname{Hilb}^{\prime}(U)(S),
$$

which induce the same object in $H_{i l b^{\prime}}(X)(S)$. Applying $\alpha$ then yields the following object in $\operatorname{Hilb}^{\prime}\left(U \times_{X} \mathcal{X}\right)(S)$ :

$\mathcal{O}_{U \times X_{X} \mathcal{X} \times S} \rightarrow\left(\pi_{U} \times i d\right)^{*}\left(\pi_{U} \times i d\right)_{*} P \otimes_{\left(\pi_{U} \times i d\right)^{*}\left(\pi_{U} \times i d\right)_{*}(\phi \times i d)^{*}(\phi \times i d)_{*} P}(\phi \times i d)^{*}(\phi \times i d)_{*} P$.

Since the adjunction morphism

$$
(\phi \times i d)^{*}(\phi \times i d)_{*} P \rightarrow P
$$

is an isomorphism, we conclude that this is the object $\mathcal{O}_{U \times{ }_{X} \mathcal{X} \times S} \rightarrow P$ we start with.

It can be seen from the proof that the universal object on $\operatorname{Hilb}(\mathcal{X})$ pulls back to the universal object on $\operatorname{Hilb}\left(U \times_{X} \mathcal{X}\right)$.

Lemma 2.2 and Proposition 2.3 imply in particular that in fact those G-Hilbert schemes patch together nicely to a global object.

\section{Proof of 1.1}

In this section we present two proofs of Theorem 1.1.

3.1. Some basic materials. We present several results concerning derived categories of coherent sheaves on smooth Deligne-Mumford stacks.

Proposition 3.1 (Serre functor). Let $\mathcal{X}$ be a smooth separated Deligne-Mumford stack which has a coarse moduli space $X$ which is a quasi-projective Gorenstein variety, whose dualizing sheaf is denoted by $\omega_{X}$. Then $D^{b}(\mathcal{X})$ has a Serre functor $S(-):=\left(-\stackrel{\mathbf{L}}{\otimes} \pi^{*} \omega_{X}\right)[\operatorname{dim} \mathcal{X}]$.

Proof. Note that $S$ is clearly an equivalence. We need to show that for $u, v \in D^{b}(\mathcal{X})$ there is a bifunctorial isomorphism

$$
\operatorname{Hom}(u, v) \simeq \operatorname{Hom}(v, S(u))^{\vee} .
$$

Our argument is parallel to that in [Ka02], Proposition 2.6. First assume that $u$ is a locally free sheaf and $v$ is a sheaf with compact support. We may assume that $u=\mathcal{O}_{\mathcal{X}}$ by replacing $v$ by $u^{\vee} \otimes v$. Now we have

$$
\operatorname{Hom}(u, v[k]) \simeq H^{k}(\mathcal{X}, v) \simeq H^{k}\left(X, \pi_{*} v\right) .
$$

On the other hand,

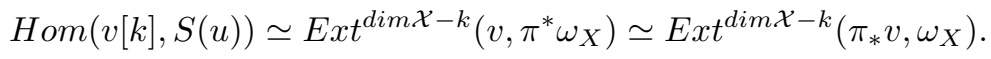

Hence $\operatorname{Hom}(u, v[k]) \simeq \operatorname{Hom}(v[k], S(u))^{\vee}$ by the duality for $X$.

If $v$ is a locally free sheaf and $u$ is a sheaf with compact support, then by the previous case, we have

$$
\operatorname{Hom}(u, v[k]) \simeq \operatorname{Hom}(S(u), S(v)[k]) \simeq \operatorname{Hom}(v[k], S(u))^{\vee} .
$$

The general case follows by taking locally free resolutions. 
Note that if $\mathcal{X}$ is isomorphic to $X$ in codimension 1 , then $\pi^{*} \omega_{X} \simeq \omega_{\mathcal{X}}$ as Cartier divisors. In this case the Serre functor is given by $S(-)=\left(-\stackrel{\mathbf{L}}{\otimes} \omega_{\mathcal{X}}\right)[\operatorname{dim} \mathcal{X}]$.

Proposition 3.2 (a spanning class). Let $\mathcal{X}$ be a smooth Deligne-Mumford stack which has Serre duality. Then the set

$$
\left\{\mathcal{O}_{\mathcal{Z}} \mid \mathcal{Z} \subset \mathcal{X} \text { is a closed substack, } \pi(\mathcal{Z}) \text { is a point in } X\right\}
$$

is a spanning class of $D^{b}(\mathcal{X})$.

Proof. This follows from the argument of [Br99], Example 2.2.

It follows that a Deligne-Mumford stack as in Proposition 3.1 has a spanning class given as above.

3.2. Reduction to local case: the first proof. Let $\left\{U_{i}\right\}$ be an étale cover of $X$ such that $U_{i} \times_{X} \mathcal{X} \simeq\left[M_{i} / G_{i}\right]$ for some schemes $M_{i}$ and finite groups $G_{i}$. Consider the diagram

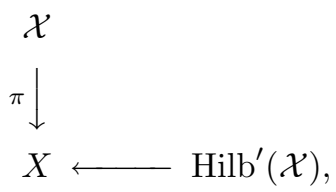

and its pullback to $U_{i}$

$$
\begin{aligned}
& \mathcal{X}_{i}:=U_{i} \times_{X} \mathcal{X} \\
& \pi_{i} \downarrow \\
& U_{i} \longleftarrow U_{i} \times_{X} \operatorname{Hilb}^{\prime}(\mathcal{X}) .
\end{aligned}
$$

Let $F: D^{b}\left(\operatorname{Hilb}^{\prime}(\mathcal{X})\right) \rightarrow D^{b}(\mathcal{X})$ be a Fourier-Mukai type transformation defined by an object $\mathcal{E} \in D^{b}\left(\operatorname{Hilb}^{\prime}(\mathcal{X}) \times \mathcal{X}\right)$ and $F_{i}: D^{b}\left(U_{i} \times_{X} \operatorname{Hilb}^{\prime}(\mathcal{X})\right) \rightarrow D^{b}\left(X_{i}\right)$ the FourierMukai type transformation given by pulling everything back to $U_{i}$.

Proposition 3.3. Assume that $F_{i}$ are equivalences of derived categories for all $i$, then so is $F$.

Proof. This follows from the argument of [Ch02], Proposition 3.2, using the spanning class given in Proposition 3.2.

Proof of Theorem 1.1. By Lemma 2.2 and Proposition 2.3, the functor

$$
F_{i}: D^{b}\left(U_{i} \times_{X} \operatorname{Hilb}^{\prime}(\mathcal{X})\right) \rightarrow D^{b}\left(\mathcal{X}_{i}\right)
$$

is the Fourier-Mukai type transformation defined by the universal object of the Hilbert scheme $\mathrm{G}_{i}$-Hilb $\left(M_{i}\right)$. By the results of [BKR01], we know that $F_{i}$ is an equivalence, $U_{i} \times_{X} \operatorname{Hilb}^{\prime}(\mathcal{X})$ is smooth, and $U_{i} \times_{X} \operatorname{Hilb}^{\prime}(\mathcal{X}) \rightarrow U_{i}$ is a crepant resolution. It follows from Proposition 2.3 that $\operatorname{Hilb}^{\prime}(\mathcal{X})$ is smooth and $\operatorname{Hilb}^{\prime}(\mathcal{X}) \rightarrow X$ is a crepant resolution. By Proposition 3.3 it follows that $F$ is an equivalence. 
3.3. Patching local crepant resolutions. In general, without knowing the global space $\operatorname{Hilb}^{\prime}(\mathcal{X})$, it is not a priori clear the crepant resolutions of $U_{i}$ given by G-Hilbert schemes can be patched to a crepant resolution of $X$. We note that it in fact can be done in dimension 3.

Proposition 3.4. Let $X$ be a normal $\mathbb{Q}$-factorial projective threefold and $\left\{U_{i}\right\}$ an open cover of $X$. Suppose that for every $i$ there is a crepant resolution $\phi_{i}: Y_{i} \rightarrow U_{i}$, then there is a crepant resolution $\phi: Y \rightarrow X$.

Proof. Consider a relative minimal model $f: Y \rightarrow X$, where $Y$ is terminal, $\mathbb{Q}$-factorial and $K_{Y}$ is $f$-nef. We only need to prove that $Y \rightarrow X$ is crepant and $Y$ is smooth. Note that $X$ has only canonical singularities since local crepant resolutions exist. The first part follows since $X$ is canonical and $K_{Y}$ is $f$-nef. To check that $Y$ is smooth we only need to check it locally. Thus we consider the restriction $\left.f\right|_{U_{i}}:\left.Y\right|_{U_{i}} \rightarrow U_{i}$. Both $\left.Y\right|_{U_{i}} \rightarrow U_{i}$ and $Y_{i} \rightarrow U_{i}$ are relative minimal models of $U_{i}$, so they can be connected by a sequence of flops. Since flops in dimension 3 preserve smoothness, the result follows.

Remark 3.5. This argument does not work in higher dimensions, since flops may not terminate and may not preserve smoothness.

3.4. Another proof of 1.1. Instead of using results of [BKR01], the second proof uses their arguments. It is not hard to check that the arguments in [BKR01] extends to our case provided Proposition 2.3 and the following:

(1) the category $D^{b}(\mathcal{X})$ is indecomposable;

(2) we have the Grothedieck duality for the morphism $\operatorname{Hilb}^{\prime}(\mathcal{X}) \times \mathcal{X} \rightarrow \operatorname{Hilb}^{\prime}(\mathcal{X})$;

(3) we have a Serre functor for $D^{b}(\mathcal{X})$.

(1) follows from the argument of [Br99], Example 3.2, provided we know that for any integral closed substack $\mathcal{W}$ of $\mathcal{X}$, the sheaf $\mathcal{O}_{\mathcal{W}}$ is indecomposible. This can be seen as follows: Since $\mathcal{X}$ is smooth, we have $\mathcal{X} \simeq\left[M / G L_{r}\right]$ where $G L_{r}$ is some general linear group. By assumption $\mathcal{X}$ is connected, so is $X$. It follows that $M$ is connected. Let $p: M \rightarrow \mathcal{X}$ be the structure morphism. Then $p^{*}: D^{b}(\mathcal{X}) \rightarrow D^{b}(M)$ is fully faithful, and $p^{*} \mathcal{O}_{\mathcal{W}}=\mathcal{O}_{\mathcal{W} \times \mathcal{X} M}$ is indecomposible. Hence $\mathcal{O}_{\mathcal{W}}$ is indecomposible.

For $(2)$, note that $\operatorname{Hilb}^{\prime}(\mathcal{X}) \times \mathcal{X} \rightarrow \operatorname{Hilb}^{\prime}(\mathcal{X})$ factors as

$$
\operatorname{Hilb}^{\prime}(\mathcal{X}) \times \mathcal{X} \stackrel{i d \times \pi}{\longrightarrow} \operatorname{Hilb}^{\prime}(\mathcal{X}) \times X \rightarrow \operatorname{Hilb}^{\prime}(\mathcal{X}) .
$$

Also note that $(i d \times \pi)_{*}$ is exact and has the left and right adjoint $(i d \times \pi)^{*}$. We may conclude by the Grothendick duality for schemes.

(3) follows from Proposition 3.1.

\section{Twisted derived McKay correspondence}

In this section we discuss the twisted version of derived McKay correspondence proposed in [BP05]. Let $X=M / G$ with $M$ quasi-projective of dimension $n$ and $G$ finite and $\mathcal{X}$ be the associated stack as discussed above. Also let $Y$ be the component of $G$-Hilbert scheme of $M$ which contains free orbits. According to [BKR01], we have an integral functor $\Phi: D^{b}(Y) \rightarrow D^{b}(\mathcal{X})$ given by the kernel $\mathcal{O}_{\mathcal{Z}}$, which is an equivalence under certain conditions. Here $\mathcal{Z}=[Z / G]$ and $Z \subset Y \times M$ is the universal closed subscheme. 
The authors of [BP05] proposed a twisted version of this equivalence, which we recast in our setting as follows: Let $B r(Y)$ be the Brauer group of $Y$. It is shown in [BP05] that both $\operatorname{Br}(Y)$ and $H^{2}\left(G, \mathbb{C}^{*}\right)$ can be embedded into a larger group, namely $\operatorname{Br}\left(X_{s m}\right)$. Let $\alpha \in \operatorname{Br}(Y) \cap H^{2}\left(G, \mathbb{C}^{*}\right)$ be a class which is $r$-torsion. It follows that there is an injective homomorphism $\iota: \mu_{r} \rightarrow \mathbb{C}^{*}$ such that $\alpha$ is in the images of the induced maps $H_{e t}^{2}\left(Y, \mu_{r}\right) \rightarrow H_{e t}^{2}\left(Y, \mathbb{C}^{*}\right)$ and $H^{2}\left(G, \mu_{r}\right) \rightarrow H^{2}\left(G, \mathbb{C}^{*}\right)$. Thus we can associate a $\mu_{r}$-gerbe $\mathcal{Y}_{\alpha}$ over $Y$ and a $\mu_{r}$-gerbe $\mathcal{X}_{\alpha}$ over $\mathcal{X}$. The $\alpha$-twisted derived categories $D^{b}(Y, \alpha)$ and $D^{b}(\mathcal{X}, \alpha)$ are derived categories of coherent sheaves on $\mathcal{Y}_{\alpha}$ and $\mathcal{X}_{\alpha}$ whose actions by $\mathbb{C}^{*}$ and $\mu_{r}$ are compatible via $\iota: \mu_{r} \rightarrow \mathbb{C}^{*}$ (see [Li04]). It is conjectured in [BP05] that there is a equivalence of derived categories

$$
D^{b}(Y, \alpha) \rightarrow D^{b}(\mathcal{X}, \alpha)
$$

We prove this conjecture in our situation. Consider the following diagram:

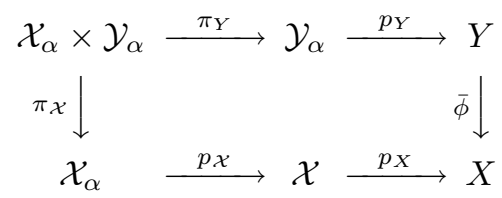

Since $\alpha \in B r(Y) \cap H^{2}\left(G, \mathbb{C}^{*}\right)$, it is not hard to check that the integral functor $\Phi_{\alpha}$ defined by the kernel $\left(p_{Y} \times p_{\mathcal{X}}\right)^{*} \mathcal{O}_{\mathcal{Z}}$ is a functor between $D^{b}(Y, \alpha)$ and $D^{b}(\mathcal{X}, \alpha)$.

Theorem 4.1. Assume that $\operatorname{dim} Y \times_{X} Y \leq n+1$, then $\Phi_{\alpha}$ is an equivalence of triangulated categories.

Proof. According to [BKR01], $Y$ is smooth and $Y \rightarrow X$ is a crepant resolution. It follows that $\mathcal{Y}_{\alpha}$ and $\mathcal{X}_{\alpha}$ are smooth Deligne-Mumford stacks. In view of this, that $\Phi_{\alpha}$ is an equivalence is a special case of a stack version of Bridgeland's result ([Br99], Theorem 1.1). It is straightforward (although lengthy) to modify Bridgeland's arguments in [Br99] to prove that $\Phi_{\alpha}$ is an equivalence if and only if

$$
\begin{gathered}
\operatorname{Ext}_{\mathcal{X}_{\alpha}}^{i}\left(\left.\left(p_{Y} \times p_{\mathcal{X}}\right)^{*} \mathcal{O}_{\mathcal{Z}}\right|_{y_{1}},\left.\left(p_{Y} \times p_{\mathcal{X}}\right)^{*} \mathcal{O}_{\mathcal{Z}}\right|_{y_{2}}\right)=0 \text { for all } i \geq 0, y_{1} \neq y_{2} \in \mathcal{Y}_{\alpha} \\
\left.\left.\left(p_{Y} \times p_{\mathcal{X}}\right)^{*} \mathcal{O}_{\mathcal{Z}}\right|_{y} \simeq\left(p_{Y} \times p_{\mathcal{X}}\right)^{*} \mathcal{O}_{\mathcal{Z}}\right|_{y} \otimes p_{\mathcal{X}}^{*} p_{X}^{*} \omega_{X} \text { for all } y \in \mathcal{Y}_{\alpha} .
\end{gathered}
$$

We simply note that in order for the arguments in [Br99] to work for this, we need to have a Serre functor, a spanning class and indecomposability of the derived categories, as well as calculations of certain Ext groups. These have been settled in Proposition 3.1, 3.2 and in Section 3.4. The needed calculations of Ext groups follows from those in [BKR01]. Now the calculations in [BKR01] immediately imply (4.1) and (4.2). Hence $\Phi_{\alpha}$ is an equivalence.

\section{Remark 4.2 .}

(1) It is natural to ask for a global version of the conjecture in [BP05]. One expects that the work of [BP05] can be generalized to give such a conjecture. Our work should be helpful in proving such a conjecture on global twisted derived McKay correspondence. 
(2) Consider the following situation: $X=M / G$ as above, $\mathcal{X}=[M / G]$ is the Deligne-Mumford stack as before, and $Y \rightarrow X$ be a crepant resolution. Suppose that there is an equivalence of derived categories $D^{b}(Y) \simeq D^{b}(\mathcal{X})$. It is known that such an equivalence is a Fourier-Mukai transform given by an integral kernel $\mathcal{E} \in D^{b}(Y \times \mathcal{X})$. If $\mathcal{E}$ is a sheaf on $Y \times \mathcal{X}$, then our arguments can be modified to show that for $\alpha \in \operatorname{Br}(Y) \cap H^{2}\left(G, \mathbb{C}^{*}\right)$, the integral functor given by the pullback of $\mathcal{E}$ to the associated gerbe yields an equivalence $D^{b}(Y, \alpha) \simeq D^{b}(\mathcal{X}, \alpha)$. It is interesting to understand whether this is the case or not, if $\mathcal{E}$ is not a sheaf.

\section{References}

[BP05] V. Baranovsky and T. Petrov, Brauer groups and crepant resolutions, Adv. Math. 209 (2007), no. 2, 547-560.

[BK90] A. Bondal and M. Kapranov, Enhanced triangulated categories, Math. USSR-Sb. 70 (1991), no. 1, 93-107.

[Br99] T. Bridgeland, Equivalences of triangulated categories and Fourier-Mukai transforms, Bull. London Math. Soc. 31 (1999), no. 1, 25-34.

[BKR01] T. Bridgeland, A. King and M. Reid, The McKay correspondence as an equivalence of derived categories, J. Amer. Math. Soc. 14 (2001), no. 3, 535-554.

[Ca00] A. Caldararu, Derived categories of twisted sheaves on Calabi-Yau manifolds, Ph.D. Thesis, Cornell University, 2000.

[Ch02] J.-C. Chen, Flops and equivalences of derived categories for threefolds with only terminal Gorenstein singularities, J. Differential Geom. 61 (2002), no. 2, 227-261.

[CT05] J.-C. Chen and H.-H. Tseng, Cone Theorem via Deligne-Mumford stacks, preprint, math.AG/0505043.

[Co05] K. Costello, The Gromov-Witten potential associated to a TCFT, preprint, math.QA/0509264.

[CI05] A. Craw and A. Ishii, Flops of G-Hilb and equivalences of derived categories by variation of GIT quotient, Duke Math. J. 124 (2004), no. 2, 259-307.

[Gr62] A. Grothendieck, Fondements de la géométrie algébrique. [Extraits du Seminaire Bourbaki, 1957-1962.] (French), Secrétariat mathématique, 1962.

[Kal05] D. Kaledin, Derived equivalences by quantization, preprint, math.AG/0504584, to appear in GAFA.

[Ka02] Y. Kawamata, Francia's flip and derived categories, Algebraic geometry, 197-215, de Gruyter, Berlin, 2002.

[Ke99] B. Keller, On the cyclic homology of exact categories, J. Pure Appl. Algebra 136 (1999), no. $1,1-56$.

[LMB00] G. Laumon and L. Moret-Bailly, Champs algebriques, Springer-Verlag, 2000.

[Li04] M. Lieblich, Moduli of twisted sheaves, Duke Math. J. 138 (2007), no. 1, 23-118.

[Ne96] A. Neeman, The Grothendieck duality theorem via Bousfield's techniques and Brown representability, J. Amer. Math. Soc. 9 (1996), no. 1, 205-236.

[OS03] M. Olsson and J. Starr, Quot functors for Deligne-Mumford stacks, Comm. Algebra 31 (2003), no. 8, 4069-4096.

[Vi89] A. Vistoli, Intersection theory on algebraic stacks and on their moduli spaces Invent. Math. 97 (1989), no. 3, 613-670.

Department of Mathematics and National Center for Theoretical Sciences,, Math. Division, Third General Bullding, National Tsing Hua University, No. 101 Sec 2 Kuang Fu Road, Hsinchu, Taiwan 30043, Taiwan

E-mail address: jcchen@math.nthu.edu, jcchenster@gmail.com 
Department of Mathematics, University of Wisconsin-Madison, Van Vleck Hall, 480 Lincoln Drive, Madison, WI 53706-1388, USA

E-mail address: tseng@math.wisc.edu 